\title{
Determinantes de saúde mental e abuso de substâncias psicoativas associadas ao tabagismo. Estudo de caso controle
}

\author{
Determinants of mental health and abuse of psychoactive \\ substances associated with tobacco use. A case-control study
}

Thiago Aquino de Amorim (https://orcid.org/0000-0002-0958-7739) ${ }^{1}$

Roselma Lucchese (https://orcid.org/0000-0001-6722-2191) ${ }^{1}$

Ernestina Maria da Silva Neta (https://orcid.org/0000-0001-5604-7287) ${ }^{2}$

Jaqueline Soares dos Santos (https://orcid.org/0000-0002-2826-3457) ${ }^{2}$

Ivânia Vera (https://orcid.org/0000-0002-8974-7949) ${ }^{1}$

Núbia Inocêncio de Paula (http://orcid.org/0000-0002-0122-5178) ${ }^{2}$

Naiane Dias Simões (http://orcid.org/0000-0002-4803-4811) ${ }^{1}$

Luiz Henrique Batista Monteiro (http://orcid.org/0000-0001-5705-3195) ${ }^{3}$

${ }^{1}$ Instituto de Biotecnologia, Universidade Federal de Goiás. Av. Lamartine P. de Avelar 1120, Campus Universitário. 75704020 Catalão GO Brasil. thiagoaquinomed@

yahoo.com.br

${ }^{2}$ Departamento de

Enfermagem, Universidade Federal de Goiás. Catalão GO Brasil.

${ }^{3}$ Faculdade de Ciências Biológicas e da Saúde, Universidade Federal dos Vales do Jequitinhonha e Mucuri. Diamantina MG Brasil.

\begin{abstract}
This study aimed to estimate the strength of association among smokers with variables regarding mental health, family functionality, and use of other psychoactive substances. This is a case-control observational study developed from March to November 2016. The study was conducted in a Brazilian Midwest municipality with 646 subjects, of which 323 were cases. In the model, the case group comprised subjects with a certain degree of tobacco dependence. The control group included subjects that were not exposed to tobacco. Concerning tobacco use time, the mean exposure of the case group was 25.65 years. In the multiple logistic regression analysis model the following were positively associated: non-white skin color $(p=0.002)$; years of study $\leq 8(p<0.001)$; having children $(p<0.001)$; informal work $(p=$ $0.024)$; not having a health plan ( $p<0.001)$; high family dysfunction ( $p=0.007)$; AUDIT $\geq 8(p<$ $0.001)$; depression ( $p<0.001)$; having illicit drug in lifetime $(p<0.001)$; living with other people ( $p$ $=0.003)$ and no religion $(p=0.001)$. This study reinforces the vulnerability of smokers, associating variables correlated to the field of mental health, and innovates by discussing the influence of family relationships on nicotinic dependence.

Key words Tobacco, Family relationships, Mental disorders, Ethnic groups, Illicit drugs
\end{abstract}

Resumo O objetivo deste estudo foi estimar a força de associação entre tabagistas com variáveis referentes à saúde mental, à funcionalidade $f a-$ miliar e ao uso de outras substâncias psicoativas. Estudo caso-controle, observacional, desenvolvido nos meses de março a novembro de 2016. O campo de estudo foi um município no Centro-Oeste do país e constituiu-se por uma população de 646 indivíduos, sendo 323 casos. No modelo, o grupo caso compreendeu indivíduos com algum grau de dependência ao tabaco. O grupo controle envolveu indivíduos que não foram expostos ao tabaco. Em relação ao tempo de tabagismo, a média de exposição do grupo caso foi de 25,65 anos. No modelo de análise múltipla de regressão logística associaram-se positivamente cor de pele não branca ( $p=$ $0,002)$; anos de estudo $\leq 8$ ( $p<0,001)$; ter filhos ( $p$ $<0,001)$; trabalho informal $(p=0,024)$; não possuir plano de saúde $(p<0,001)$; elevada disfunção familiar ( $p=0,007)$; AUDIT $\geq 8(p<0,001)$; depressão ( $p<0,001)$; já ter usado droga ilícita na vida ( $p<0,001)$; morar com outras pessoas ( $p$ $=0,003)$ e não possuir religião $(p=0,001)$. Este estudo reforça a vulnerabilidade dos fumantes investigados, ao associar variáveis correlacionadas ao âmbito da saúde mental, e inova ao discutir a influência das relações familiares na dependência nicotínica.

Palavras-chave Tabaco, Relações familiares, Transtornos mentais, Grupos étnicos, Drogas ilícitas. 


\section{Introdução}

Há um consenso mundial de que a dependência da nicotina é um grave problema de saúde pública, cuja mortalidade anual é de aproximadamente 5,4 milhões de pessoas, superior quando comparada à letalidade por doenças infectocontagiosas, como a AIDS, tuberculose e malária ${ }^{1}$. O tabaco é a principal causa de morte evitável no mundo, com estimativa ascendente até 2030 de aproximadamente 8 milhões de óbitos ao ano 2 . No Brasil, o número de fumantes tem se reduzido desde a década de 1990, mas estima-se que a população de fumantes seja de 27,9 milhões, e que o tabagismo cause 200 mil óbitos anualmente ${ }^{1}$.

De acordo com a décima revisão do Código

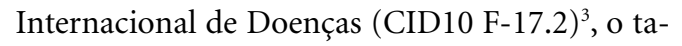
bagismo possui potencial para ocasionar dependência da nicotina, e desencadear transtornos mentais e comportamentais, dentre os quais sintomas depressivos, transtorno bipolar, ansiedade, transtorno da personalidade e de défice de atenção. Os fumantes que apresentam dependência da nicotina têm de 2,7 a 8,1 vezes mais probabilidade de desenvolver estes transtornos, respectivamente, quando comparados com fumantes esporádicos, ex-fumantes e indivíduos que nunca fumaram ${ }^{4}$. Assim, o tabagismo se classifica como uma doença crônica ${ }^{3}$, associada à diversos sintomas, que pode comprometer substancialmente as condições de saúde 4 .

O tabagismo é uma causa evitável de vários agravos crônicos à saúde, associados às neoplasias, doenças cardiovasculares, pulmonares, alterações oftalmológicas, entre outras ${ }^{5}$. Em meio a essas comorbidades, a associação com outras variáveis são apontadas por pesquisadores, como: sexo masculino $0^{6}$, idade adulta ${ }^{7}$, condições econômicas desfavoráveis ${ }^{8}$, situação de desemprego, baixo nível de escolaridade ${ }^{6,8-10}$, baixo escore do índice de massa corporal (IMC), o consumo atual de bebidas alcoólicas ${ }^{11}$ e residir em zonas rurais ${ }^{8,12}$.

$\mathrm{O}$ contexto das relações familiares, em particular a disfuncionalidade familiar, é um importante fator de risco para o desenvolvimento de dependência de substâncias psicoativas. Alguns estudos realizados com adolescentes têm demostrando que conviver em um ambiente familiar disfuncional está associado com o aumento da probabilidade de se tornar tabagista. Embora as consequências da dependência nicotínica apresentem impactos relevantes no âmbito mundial, investigações referentes à influência das relações familiares no consumo do tabaco ainda são insuficientes ${ }^{13}$.
No entanto, consideram-se, para este estudo, as associações com outras morbidades, no campo da saúde mental, e/ou comportamentos que agravariam ainda mais a condição de transtornos ocasionados pelo uso recorrente de tabaco. Diante do exposto, tem-se por hipótese que as comorbidades psiquiátricas e o uso de outras substâncias psicoativas associam-se à dependência ao tabaco, condição que potencializa os agravos crônicos à saúde e que impacta nas relações destes indivíduos, sobretudo a familiar.

Este estudo inovou ao testar a variável funcionalidade familiar em meio à condição de vulnerabilidade à qual estão expostos indivíduos com níveis de dependência nicotínica. Para tanto, objetivou-se estimar a força de associação entre características do grupo tabagista com variáveis referentes à saúde mental, à funcionalidade familiar e ao uso de outras substâncias psicoativas.

\section{Método}

Trata-se de um estudo caso-controle, com caráter observacional, empregado para investigar características que ocorrem com maior ou menor frequência no grupo caso, quando comparado ao controle ${ }^{14}$. No modelo, o grupo caso compreendeu os indivíduos com algum grau de dependência do tabaco, e o grupo controle envolveu os indivíduos que não foram expostos ao tabaco. $\mathrm{O}$ campo de estudo deu-se em um município de relevante papel econômico e social no Centro-Oeste do país.

A população para o grupo caso foi fumante regular (últimos 30 dias $^{15}$ ) e, para controle, indivíduos não fumantes, ambos moradores no município em questão. O cálculo amostral foi realizado no programa Stata Software Package (STATA), versão 14.0, utilizando-se os comandos sampsi 15.25 , power $(.80) \mathrm{a}(.05)^{16}$, considerando $15 \%$ de prevalência de tabagismo regular na população brasileira ${ }^{5}$, aumento hipotético de $25 \%$ para os problemas relacionados ao consumo de tabaco (aplicado para caso), poder de teste de $80 \%$ e nível de significância de $5 \%$. Ao resultado obtido, somaram-se $20 \%$, prevenindo-se perdas, resultando para $\mathrm{n} 1$ (controle) 322 indivíduos e, para n2 (caso), 323 indivíduos, totalizando 645 indivíduos para serem entrevistados.

Toda a amostra foi pareada por sexo e idade, para controle de possível viés de confusão ${ }^{14}$. Como critério de elegibilidade de casos, foram estipulados ter idade $\geq 17$ anos, ser morador na cidade onde a pesquisa foi desenvolvida e ser fu- 
mante regular por tempo $\geq 12$ meses, resultado menos ou igual ao grau mínimo de dependência ao tabaco mensurado pelo instrumento Fagerström Test For Nicotine Dependence (FTND) ${ }^{17}$. Os não elegíveis, tanto para o caso, como para o controle, foram os não fumantes que relataram doenças cardiovasculares congênitas sem correção e diagnóstico médico de transtorno mental de natureza psicótica.

A amostra para o grupo controle foi orientada pelos critérios de elegibilidade: $\geq 17$ anos, residir no mesmo município da realização da pesquisa, nunca ter fumado na vida (não ser fumante atual e/ou ex-fumante) e não ter convivência domiciliar com fumantes. Excluíram-se não fumantes que autorreferiram ter problemas cardiovasculares congênitos sem correção, e que apresentaram diagnóstico psiquiátrico de transtorno mental grave e persistente (com sintomas psicóticos).

Recrutaram-se indivíduos para o grupo caso por conveniência, de acordo com os critérios de elegibilidade em cenários conveniados com a universidade para prática de ensino, extensão e pesquisa. Eram eles comunidade (domicílios, espaços acadêmicos e sociais), dispositivos de Atenção Primária à Saúde (Unidades Básicas de Saúde e Estratégia Saúde da Família), de urgência e emergência (unidade de pronto atendimento), e outros de Atenção especializada e média complexidade (hospitais e centros de testagem e aconselhamento). Como controle, aplicou-se o critério de vizinhança ${ }^{14}$. Os indivíduos não fumantes foram convidados nas localidades em que se abordou um fumante, mantendo-se proporção semelhante entre os dois grupos.

$\mathrm{O}$ teste piloto constituiu um ensaio que reproduziu todas as estratégias e métodos que seriam utilizados no estudo, aplicado a dez indivíduos (cinco fumantes e cinco não fumantes) que estavam no município onde o estudo foi realizado, porém não residiam ali. A aplicação do questionário na versão piloto objetivou treinar a equipe de pesquisadores de campo e identificar os aspectos logísticos e operacionais, a fim de ajustar o instrumento de coleta de dados. Estas informações não entraram na análise final dos dados.

Os dados foram coletados nos meses de março a novembro do ano de 2016 por pesquisadores de campo, estudantes de graduação de curso na área da saúde devidamente treinados.

As entrevistas foram face a face, respeitandose a disponibilidade de cada indivíduo, em local reservado. Nesse momento, receberam orienta- ções acerca do estudo e assinaram o Termo de Consentimento Livre e Esclarecido (TCLE) para os indivíduos com idade $\geq 18$ anos ou Termo de Assentimento Livre e Esclarecido (TALE) para aqueles com idade de 17 anos completos.

O instrumento com dados sociodemográficos e históricos clínicos foi elaborado pelos pesquisadores orientados pelos referenciais na saúde mental ${ }^{4}$. Para seleção do grupo caso e descrição do padrão de dependência nicotínica, aplicou-se o FTND ${ }^{17,18}$, com seis questões que rastreavam a dependência nicotínica. Os escores de zero a 2 pontos refereriram-se a muito baixa dependência; de 3 a 4, a baixa dependência; 5, média dependência; de 6 a 7, elevada dependência; e de 8 a 10 pontos, muito elevada dependência.

Para a mensuração da funcionalidade familiar, utilizou-se o instrumento APGAR de família, que, a partir da pessoa índice, permite avaliar cinco aspectos: adaptation (adaptação), partnership (companheirismo), growth (desenvolvimento), affection (afetividade) e resolve (capacidade resolutiva). Cada letra inicial recebe pontuação de zero a 2 pontos. A elevada disfunção familiar (EDF) é caracterizada pela pontuação de zero a 4 pontos; 5 a 6 pontos indicam moderada disfunção familiar (MDF); de 7 a 10 pontos, trata-se de boa funcionalidade familiar (BFF). O instrumento APGAR de família foi desenvolvido por Smilkstein, em $1978^{19}$. Sua validação no Brasil foi realizada por meio da tradução e da adaptação transcultural, com participação de oito juízes, em 2001, além de ter sido feita uma análise psicométrica, por estudo descritivo, de campo e transversal, com população de idosos ${ }^{20}$. Para determinar o padrão de uso de risco de álcool, utilizou-se o instrumento Alcohol Use Disorder Identification Test (AUDIT), validado no Brasil em $1999^{21}$ como forma de rastreio para detecção precoce do uso de álcool, composto por dez questões, em que a pontuação máxima é 40 pontos. Adotaram-se $\geq$ 8 pontos como ponto de corte ${ }^{22}$. Para os instrumentos APGAR, FTND e AUDIT, realizou-se teste de confiabilidade alfa de Cronbach.

As variáveis foram de dois níveis: I (indivíduo) e II (contexto). No nível I, o pareamento da amostra deu-se por sexo (feminino vs. masculino) e categoria idade ( $<30$ vs. 30 a 39 vs. 40 a 49 vs. 50 a 59 vs. $\geq 60$ ). As variáveis independentes foram cor da pele autorreferida (branca vs. não branca), anos de estudo ( $\leq 8$ anos vs. $>8$ anos); ter filhos (sim vs. não); estado civil (viver sem companheiro vs. viver com companheiro); trabalho formal (regime de trabalho segundo a legislação nacional) vs. informal (autônomo ou 
desempregado); possui religião (autorreferida sim vs. não); não possui plano de saúde (sim vs. não); morar com familiares, de acordo com o próprio conceito de família vs. outras pessoas; EDF - escore $\leq 4$ pelo APGAR de família (não vs. sim); depressão - tem ou já teve diagnóstico médico de depressão (não vs. sim); já usou drogas ilícitas - já fez uso de drogas como maconha, cocaína, crack, dietilamida do ácido lisérgico (LSD) ou inalantes de comercialização ilegal (não vs. sim); AUDIT (escore $<8$ vs. $\geq 8$ ) e diagnóstico de doença isquêmica do coração - autorreferida por diagnóstico médico (sim vs. não).

Nas variáveis de nível II, as contextuais foram inseridas no modelo de análise multinível e orientadas pela importância no controle de confusã ${ }^{23}$, uma vez que situações de doença ou saúde relacionam-se com fatores contextuais. A compreensão do processo saúde-doença deve se fundamentar no intercâmbio entre indivíduo e contexto $^{24}$. Estas variáveis foram comunidade vs. serviços de Atenção Primária à Saúde vs. hospitais e serviços de especialidade vs. rede de urgência com efeito na análise múltipla (conforme a localidade de abordagem do caso e controle). Foi covariável na análise do nível II Atenção Primária vs. Atenção Secundária (atendendo ao nível de complexidade de atenção à saúde correspondente às quatro categorias da variável contexto).

Para análise estatística das variáveis associadas ao grupo, utilizou-se o software STATA 14.0 e, como medida de associação, o odds ratio (OR), considerando o intervalo de confiança de $95 \%$ (IC95\%). Pela obtenção da OR bruta, escolheram-se as variáveis com $\mathrm{p}<0,10$ para o modelo de regressão logística multinível. No nível I as variáveis de indivíduo foram inseridas seguindo o modelo teórico de inserção sempre ajustado pelo nível II (variável e covariável contextuais), representação gráfica na Figura 1.
A qualidade de ajuste entre os modelos foi avaliada por meio do teste de-loglikelihood, e pelos valores da variance do nível II, do coeficiente de erro padrão (CE) desvio do erro padrão (SE).

$\mathrm{O}$ estudo foi desenvolvido respeitando-se os princípios éticos que norteiam as pesquisas com seres humanos no Brasil ${ }^{25}$ e ocorreu após autorização do Comitê de Ética em Pesquisa (CEP).

\section{Resultados}

O estudo contou com amostras de 322 participantes no grupo controle e 323 grupo caso. As perdas amostrais foram repostas pelos pesquisadores, resguardando o cálculo amostral e o pareamento por categoria idade e sexo. O grupo controle constituiu-se de indivíduos com média de idade de 40,22 anos (desvio padrão - DP de $14,74)$ enquanto que, no grupo caso, a média de idade foi de 40,88 anos (DP de 13,21). Quanto ao tempo de tabagismo, a média de exposição do grupo caso foi de 25,65 anos (DP de 13,87). Na Tabela 1, estão expostas as informações das categorias de pareamento da amostra.

Nas Tabelas 2 e 3, observa-se a OR bruta e ajustada. Cabe salientar que a OR ajustada foi realizada no modelo com o fator contextual.

$\mathrm{Na}$ análise bivariada associaram-se positivamente as variáveis: cor de pele não branca $(\mathrm{p}<$ 0,001 ; OR 2,65; IC95\% 1,86-3,79); anos de estudo $\leq 8(\mathrm{p}<0,001$; OR 3,93; IC95\% 2,79-5,52); ter filhos ( $\mathrm{p}<0,001$; OR 2,22; IC95\% 1,56-3,16); viver com companheiro $(\mathrm{p}=0,018$; OR 1,45 ; IC95\% 1,06-1,98); trabalho informal ( $\mathrm{p}<0,001$; OR 1,73; IC95\% 1,11-2,70); não possui plano de saúde ( $\mathrm{p}<0,001$; OR 3,79; IC95\% 2,63-5,46); EDF ( $<<0,001$; OR 3,20; IC95\% 1,87-5,47); AUDIT $\geq 8$ (p < 0,001; OR 4,94; IC95\% 3,30-7,38); depressão ( $p<0,001$; OR 4,15; IC95\% 2,33-

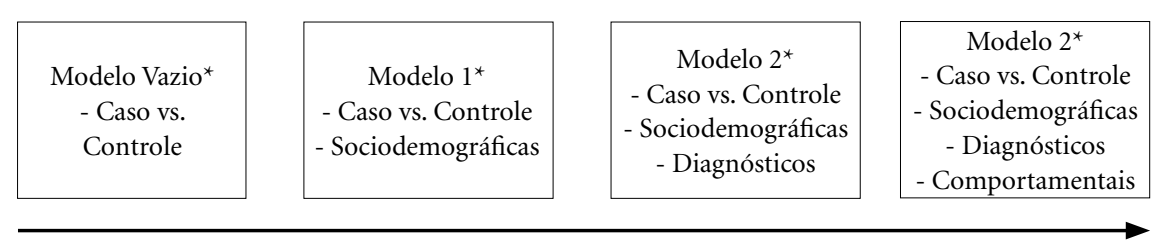

Figura 1. Modelo conceitual da análise multinível. 
Tabela 1. Dados de pareamento da amostra caso e controle. Centro-Oeste, 2016.

\begin{tabular}{lcccc}
\hline \multirow{2}{*}{ Variáveis categóricas } & \multicolumn{2}{c}{ Controle $(\mathbf{n}=\mathbf{3 2 2})$} & \multicolumn{2}{c}{ Caso $(\mathbf{n}=\mathbf{3 2 3})$} \\
\cline { 2 - 5 } & $\mathbf{N}$ & $\mathbf{\%}$ & $\mathbf{N}$ & $\mathbf{\%}$ \\
\hline Sexo & & & & \\
$\quad$ Feminino & 175 & 54,9 & 175 & 53,9 \\
$\quad$ Masculino & $145^{*}$ & 45,1 & 148 & 46,1 \\
Idade & & & \\
$\quad<30$ & 67 & 21,0 & 67 & 20,7 \\
$30-39$ & 93 & 29,2 & 94 & 29,1 \\
$40-49$ & 85 & 26,6 & 85 & 26,3 \\
$50-59$ & 40 & 12,5 & 44 & 13,6 \\
$\geq 60$ & $34^{*}$ & 10,7 & 33 & 10,6 \\
Variável contextual & & & & \\
Urgência & 88 & 49,16 & 91 & 50,84 \\
Comunidade & 105 & 55,56 & 84 & 44,44 \\
Atenção Primária & 55 & 45,86 & 65 & 57,17 \\
Hospitais e serviços de especialidade & $72^{*}$ & 46,45 & 83 & 53,55 \\
\hline
\end{tabular}

Número válido para casela.

$7,40)$; já ter usado droga ilícita na vida ( $\mathrm{p}<0,001$; OR 7,37; IC95\% 4,15-13,10); morar com outras pessoas ( $\mathrm{p}<0,001$; OR 2,39; IC95\% 1,56-3,65); e não possuir religião ( $<<0,001$; OR 2,52; IC95\% 1,61-3,96).

Após a análise múltipla de regressão logística com fator contextual de local de entrevista, permaneceram associadas positivamente as variáveis: cor de pele não branca ( $\mathrm{p}=0,002$; OR 2,08; IC 95\% 1,31-3,32); anos de estudo $\leq 8$ ( $\mathrm{p}<$ 0,001; OR 3,41; IC95\% 2,19-5,31); ter filhos ( $<<$ 0,001; OR 3,31; IC95\% 1,87-5,86); trabalho informal ( $\mathrm{p}=0,024$; OR 1,97; IC95\% 1,09-3,55); não possuir plano de saúde $(\mathrm{p}<0,001$; OR 2,92; IC95\% 1,82-4,68); EDF ( $\mathrm{p}=0,007$; OR 2,70; IC95\% 1,31-5,55); AUDIT $\geq 8$ ( $<<0,001$; OR 4,81; IC95\% 2,83-8,18); depressão ( $<<0,001$; OR 3,77; IC95\% 1,88-7,55); já ter usado droga ilícita na vida ( $<$ 0,001; OR 7,66; IC95\% 3,61$16,25)$; morar com outras pessoas ( $\mathrm{p}=0,003$; OR 2,42; IC95\% 1,36-4,31); e não possuir religião (p $<$ 0,001; OR 2,86; IC95\% 1,54-5,29).

$\mathrm{Na}$ Tabela 3, notou-se a redução do teste -2 loglikelihood na medida que os modelos foram inseridos, conferindo à análise melhor qualidade de ajuste conforme se inseriram os grupos de variáveis individuais; a melhor qualidade da variação pelo ajuste do nível II está expressa no valor maior que o dobro do coeficiente erro padrão em relação ao desvio erro padrão no modelo final. No estudo múltiplo, peraneceram associadas ao grupo caso cor da pele não branca, anos de estudo $\leq 8$ anos, ter filhos, trabalho informal, não possui plano de saúde, referir EDF, AUDIT $\geq 8$, referir diagnóstico de depressão, já ter usado droga ilícita na vida e não possuir religião.

\section{Discussão}

O presente estudo trouxe como principal evidência a associação ao grupo tabagista de variáveis já conhecidas (tanto na dimensão sociodemográfi$\mathrm{ca}$, na de doenças mentais, como relacionadas ao uso de outras substâncias psicoativas e comportamentais) e outra ainda não investigada, relativas à funcionalidade familiar, como o escore do instrumento APGAR de avaliação para EDF, corrobora nas implicações de saúde mental da população tabagística ${ }^{26}$. Também ressalta-se a aplicação da variável contexto na análise múltipla, na busca de uma evidência mais ampliada sobre o fenômeno estudado e controle de possível viés.

Os limites da investigação relacionam-se ao delineamento da pesquisa de caso-controle, especialmente em relação à memória e à seleção do grupo controle. Na tentativa de minimizá-los, a equipe foi treinada para a abordagem e a obtenção dos dados relatados, e o indivíduo controle foi o mais representativo possível em relação a sexo, faixa etária e critério de vizinhança.

Quanto à cor da pele, os resultados sugerem que os indivíduos que se autodeclararam não brancos estavam em risco para algum nível de dependência nicotínica. Esta associação pode ser explicada devido ao tempo que o organismo de- 
Tabela 2. Análise bivariada entre grupo controle e grupo caso com variáveis independentes. Centro-Oeste, 2016.

\begin{tabular}{|c|c|c|c|c|}
\hline Variável & $\begin{array}{c}\text { Controle } \\
(\mathbf{n}=322) \\
\mathbf{n}(\%)\end{array}$ & $\begin{array}{c}\text { Caso } \\
(\mathbf{n}=323) \\
\mathbf{n}(\%)\end{array}$ & $\begin{array}{c}\text { OR bruta* } \\
\text { (IC95\%) }\end{array}$ & Valor de $p$ \\
\hline \multicolumn{5}{|l|}{ Cor de pele } \\
\hline Branca & $125(66,49)$ & $63(33,51)$ & 1 & \\
\hline Não branca & $195(42,86) \dagger$ & $260(57,14)$ & $2,65(1,86-3,79)$ & $<0,001$ \\
\hline \multicolumn{5}{|l|}{ Anos de estudo } \\
\hline$>8$ & $246(62,44)$ & $148(37,56)$ & 1 & \\
\hline$\leq 8$ & $74(29,72) \dagger$ & $175(70,28)$ & $3,93(2,79-5,52)$ & $<0,001$ \\
\hline \multicolumn{5}{|l|}{ Tem filhos } \\
\hline Não & $116(63,74)$ & $66(36,26)$ & 1 & \\
\hline Sim & $203(44,13) \dagger$ & $257(55,87)$ & $2,22(1,56-3,16)$ & $<0,001$ \\
\hline \multicolumn{5}{|c|}{ Vive com companheiro } \\
\hline Não & $169(54,34)$ & $142(45,66)$ & 1 & \\
\hline Sim & $148(44,98) \dagger$ & $181(55,02)$ & $1,45(1,06-1,98)$ & 0,018 \\
\hline \multicolumn{5}{|l|}{ Trabalho } \\
\hline Formal & $282(51,74)$ & $263(48,26)$ & 1 & \\
\hline Informal & $37(38,14) \dagger$ & $60(61,86)$ & $1,73(1,11-2,70)$ & $<0,001$ \\
\hline \multicolumn{5}{|l|}{ Plano de saúde } \\
\hline $\operatorname{Sim}$ & $137(43,77)$ & $55(17,03)$ & 1 & \\
\hline Não & $176(56,23) \dagger$ & $268(82,97)$ & $3,79(2,63-5,46)$ & $<0,001$ \\
\hline \multicolumn{5}{|l|}{ EDF } \\
\hline Não & $299(52,92)$ & $266(47,08)$ & 1 & \\
\hline Sim & $20(25,97) \dagger$ & $57(74,03)$ & $3,20(1,87-5,47)$ & $<0,001$ \\
\hline \multicolumn{5}{|l|}{ Pontuação do AUDIT } \\
\hline$<8$ & $281(59,51)$ & $191(40,47)$ & 1 & \\
\hline$\geq 8$ & $39(22,94) \dagger$ & $131(77,06) \dagger$ & $4,94(3,30-7,38)$ & $<0,001$ \\
\hline \multicolumn{5}{|l|}{ Depressão } \\
\hline Não & $304(95,00)$ & $265(82,04)$ & 1 & \\
\hline Sim & $16(5,00) \dagger$ & $58(17,96)$ & $4,15(2,33-7,40)$ & $<0,001$ \\
\hline \multicolumn{5}{|c|}{ Já usou droga ilícita na vida } \\
\hline Não & $305(56,27)$ & $237(43,73)$ & 1 & \\
\hline Sim & $15(14,85) \dagger$ & $86(85,15)$ & $7,37(4,15-13,10)$ & $<0,001$ \\
\hline \multicolumn{5}{|c|}{ Morar com outras pessoas } \\
\hline Familiares & $281(53,52)$ & $244(46,48)$ & 1 & \\
\hline Outras pessoas & $38(32,48) \dagger$ & $79(67,52)$ & $2,39(1,56-3,65)$ & $<0,001$ \\
\hline \multicolumn{5}{|l|}{ Possui religião } \\
\hline Sim & $287(53,25)$ & $252(46,75)$ & 1 & \\
\hline Não & $32(31,10) \dagger$ & $71(68,93)$ & $2,52(1,61-3,96)$ & $<0,001$ \\
\hline
\end{tabular}

manda para metabolizar a nicotina, uma vez que indivíduos brancos apresentam taxas de metabolização mais elevadas do que os indivíduos afro -americanos. Logo, estes tendem a fumar em menores quantidades ${ }^{27}$ e demoram mais a fumar o primeiro cigarro do dia ${ }^{28}$. Ambos os mecanismos impactam em escores de avaliação de dependência da nicotina, quantidade e primeiro cigarro do dia, segundo o FTND. Outro estudo realizado em uma população multirracial mostrou que o nível plasmático da cotinina (metabólito da nicotina) apresentava-se seis vezes mais elevado nos participantes afro-americanos com histórico de exposição ao tabagismo passivo, quando comparados às outras raças ${ }^{29}$.

Em consonância com estes dados da literatura internacional, estudos recentes realizados no Brasil mostram que a prevalência de fumantes 


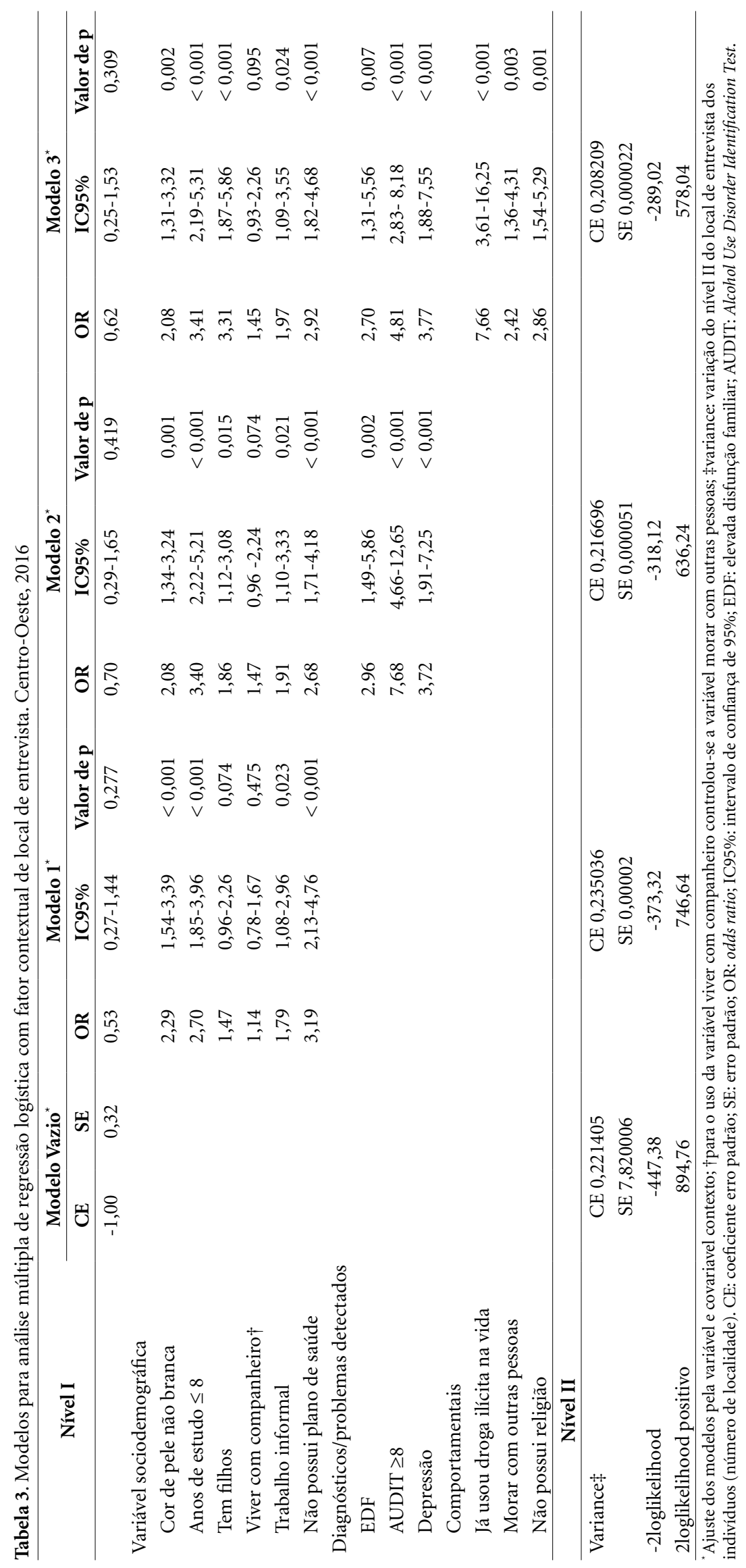


autodeclarados negros é relativamente maior do que os autodeclarados brancos ${ }^{30,31}$. Os dados do Instituto Brasileiro de Geografia e Estatística (IBGE) de 2017 corroboram este achado ao afirmarem a existência de desigualdade social entre estes grupos raciais, sendo a população negra e parda a que possui maior restrição de acesso à saúde, educação e proteção social ${ }^{32}$.

Em relação ao baixo nível de escolaridade e o trabalho informal ${ }^{10,33}$ sugere-se que os indivíduos com baixo nível de escolaridade e os trabalhadores informais estão suscetíveis a fumarem mais e com maior variedade de produtos derivados do tabaco. O uso do tabaco associado ao baixo nível de escolaridade indica que há insuficiência de conhecimento sobre os malefícios que os produtos do tabaco ocasionam no indivíduo fumante ${ }^{7,8,34}$,tanto que há evidências de que residir em áreas de difícil acesso ao ensino favorece o abuso de tabaco $^{8}$.

Quanto a variável ter filhos, esta análise apontou que os indivíduos que possuíam filhos tendiam a fumar mais. Contrariamente, na literatura, discute-se outro achado: o fato de que os filhos atuam como estratégia para encorajar a cessação do tabagismo, uma vez que pais e filhos compreendem que o tabagismo também pode afetar a saúde dos filhos futuramente ${ }^{35}$. Um outro achado também aborda que entre os indivíduos que possuem pelo menos um filho, há uma associação positiva quanto ao uso de bebidas alcoóli$\mathrm{cas}^{36}$. No entanto, a variável ter filhos requer mais estudos, pois seu papel na associação com o uso de substâncias psicoativas não está bem esclarecida na literatura.

Quanto ao status trabalho, indivíduos desempregados ou que atuam como profissionais autônomos apresentam maiores chances de consumirem tabaco quando comparados aos indivíduos que possuem trabalho formal ${ }^{37,38}$. Destes, o grupo dos profissionais autônomos apresentaram maiores chances de uso $^{39}$. Para algumas condições de trabalho, tal relação pode ser explicada por meio do controle exercido pela instituição empregadora e da imposição de regras internas antitabagismo aos trabalhadores durante o processo laboral, o que limita substancialmente o tempo dispensado para o hábito de fumar durante o período de cumprimento das horas de trabalho ${ }^{38}$. Os fatores de controle sobre o tabagismo em ambientes laborais, tanto quanto a irregularidade de atividade física e as condições econômicas, devem ser considerados em estudos futuros, para ampliação de conhecimento sobre tais aspectos relacionados com a dependência nicotínica.
Uma condição que complementa a discussão anterior é a variável não possuir plano de saúde, que foi positivamente associada ao uso do tabaco. Esta situação, inerente à condições de aquisição de serviços na área de saúde suplementar, pode se articular com as condições de trabalho com vínculo empregatício legalizado, no entanto, ainda são incipientes em estudos sobre o tabagismo. No Brasil, estudos mostram que as condições sociodemograficas estão diretamente relacionadas com o fato de possuir plano de saúde; indivíduos com baixa situação economica, baixa escolaridade, profissão informal e autodeclarados negros apresentam menor acesso aos serviços de saú$\mathrm{de}^{40,41}$. Ao se aproximar desta discussão, encontrou-se a evidência de que as pessoas atentidas nos serviços de saúde aos quais estão vinculadas podem receber orientações dos profissionais sobre os riscos relacionados ao tabagismo, além de questionarem seus hábitos e fornecerem conselhos para cessação ${ }^{31}$. Este dado implica que indivíduos que possuem planos de saúde são melhores assistidos, favorecendo a adoção de um estilo de vida saudável. Porém, essa variável requer melhor exploração para apontamentos mais contundentes.

O tabagismo, além de comprometer a saúde do indivíduo, afeta as relações familiares, o que pode originar um distanciamento entre os membros da família e comprometer a boa funcionalidade familiar (BFF). As alterações nas relações familiares ocorrem porque os fumantes passivos tendem a se sentirem estressados e desamparados no ambiente familiar, além de adotarem percepções negativas com relação ao papel desempenhado pelos parentes dentro do sistema familiar $^{42}$. O contrário também pode ser percebido, quando qualquer tipo de disfunção (leve, moderada ou grave) no sistema familiar torna os indivíduos deste grupo mais propensos ao uso de substâncias psicoativas, principalmente o tabaco $^{26,43,44}$. Estudos realizados com adolescentes corroboram este achado, ao demonstrarem que a presença de fumantes no ambiente familiar encoraja os adolescentes a fazerem uso de tabaco, seja pela reprodução do comportamento positivo ao tabagismo ou em decorrência da exposição à fumaça do cigarro, resultando em maior sensibilidade à dependência da nicotina ${ }^{43,45}$.

Ao mesmo tempo, residir com outras pessoas, além dos familiares, aumenta as chances de um indivíduo consumir tabaco e/ou outras drogas. Esta associação pode ser explicada devido ao distanciamento que ocorre em relação aos familiares, com consequente diminuição e/ou cessação 
do efeito protetor, que anteriormente inibia os comportamentos desviantes da prole, em particular o uso de substancias psicoativas ${ }^{46,47}$. Este achado evidencia a importância da boa relação familiar como um fator de prevenção do uso de substâncias ilícitas, mas discussões futuras neste sentido se fazem necessárias para esclarecer melhor esta associação.

O uso do tabaco, além de contribuir para diversas enfermidades, também pode estar associado a um maior risco de desenvolver distúrbios mentais, entre eles a depressão. Na literatura, a causalidade entre tabagismo e depressão é atribuída a mecanismos distintos, entre os quais o risco seria diretamente proporcional ao uso do tabaco, ou seja, quanto maior for o uso de produtos tabagísticos, mais elevadas seriam as probabilidades de desenvolver a doenç̧⿻ ${ }^{48,49}$. Assim, sugere-se que, devido à ação da nicotina nos sistemas neuroquímicos, bem como nas funções neuroendócrinas, ocorreria uma interferência na evolução do estado pré-patológico ${ }^{49}$. Também se tem uma causalidade inversa: indivíduos depressivos possuem 1,5 vez mais chances para iniciar e manter o uso de derivados do tabaco $0^{50}$, e isto pode ser explicado pela ausência de comportamentos para autopreservação do estado de saú$\mathrm{de}^{51} \mathrm{e}$ pelo aumento das sensações prazerosas ao fumar ${ }^{49}$.

$\mathrm{Na}$ presente investigação, houve associação negativa no grupo caso com o status ter religião. Há quase um consenso entre os estudos de que ser praticante religioso é protetor neste contexto. $\mathrm{O}$ indivíduo que possui um elevado vínculo religioso tende a apresentar menores probabilidades de iniciar e manter o consumo de produtos tabagísticos diariamente ${ }^{52-54}$, uma vez que as orientações dos preceitos religiosos reforçam positivamente o comportamento dos não fumantes $e$ encorajam os indivíduos que fazem o uso persistente de produtos tabagísticos a diminuírem e/ou cessarem este consumo ${ }^{52,55}$. Além disto, fornece aos indivíduos mecanismos de apoio emocional contra fatores estressantes, que possuem potencial para desencadear o uso de tabaco ${ }^{53}$.

Os indivíduos que fumam cigarros regularmente, quando comparados com os indivíduos que não são fumantes regulares, estão mais predispostos a iniciarem precocemente o uso de drogas ilícitas, especialmente a maconha e ainda relatarem oportunidades prévias de consumo ${ }^{56}$ Esta associação pode estar relacionada a fatores genéticos, ambientais ${ }^{57} \mathrm{e}$ à presença de condutas problemáticas, como hiperatividade, impulsividade e agressividade ${ }^{58}$.

Quanto à relação entre comorbidades do uso de álcool e outras drogas, este estudo não apresenta inovações, porém a relação de causalidade coexistente é forte. $\mathrm{O}$ tabagismo e o consumo diário de bebidas alcoólicas estão integrados. Indivíduos com comportamentos tabagísticos estão mais propensos a ingerirem álcool, do mesmo modo que os indivíduos que ingerem álcool também estão propensos a usar produtos tabagísticos; esta associação indefere do tempo de exposição ${ }^{59}$. Dentre os usuários de tabaco, aqueles que fazem consumo diário mais elevado estão associados a maiores escores do AUDIT ${ }^{60}$. Estudos sugerem que o uso concomitante da nicotina com o etanol atua reforçando as sensações prazerosas do álcool e reduzindo seus efeitos indesejados ${ }^{61}$. Também existem hipóteses em que a associação entre tabaco e álcool atuaria ativando o sistema mesolímbico dopaminérgico, que, por sua vez, agiria elevando as sensações de gratificação como reforço positivo à dependência de substâncias psicoativas $^{62}$.

\section{Conclusão}

Os resultados deste estudo indicaram associação de variáveis no âmbito da saúde mental que reforçam a vulnerabilidade dos fumantes investigados, sobretudo para os de baixa escolaridade, sem vínculo trabalhista formal e em relação ao uso de risco de álcool e drogas ilícitas. Quanto ao achado principal, a elevada disfunção familiar apresentou-se fortemente associada a algum grau de dependência nicotínica, ressalta-se que outras dimensões relacionadas à constituição famíliar podem corroborar esta situação, como ter filhos e residir com pessoa não familiares. Estas variáveis, quando presentes em um mesmo contexto, potencializam-se, aumentado a exposição às comorbidade relacionadas ao uso do tabaco e seu impacto social, econômico e saúde. Este estudo revela a necessidade de adoção de políticas públicas voltadas à atenção à saúde mais efetivas e abrangentes, em relação à dinâmica familiar. 


\section{Colaboradores}

TA Amorim e R Lucchese: concepção e planejamento do projeto de pesquisa, coleta e/ou análise e interpretação dos dados, redação do manuscrito, revisão do manuscrito; EM Silva Neta, LHB Monteiro, NI Paula e ND Simões: coleta e/ ou análise e interpretação dos dados, redação do manuscrito, revisão do manuscrito JS Santos e I Vera: concepção e planejamento do projeto de pesquisa, análise e interpretação dos dados, redação do manuscrito, revisão do manuscrito.

\section{Referências}

1. Sociedade Brasileira de Pneumologia e Tisiologia (SBPT). Diretrizes em foco: Tabagismo. Rev. Assoc. Med. Bras. 2010; 56(2):127-143. [acessado 2018 Jan 10]. Disponível em: http://www.scielo.br/scielo. php?script $=$ sci_arttext\&pid $=$ S0104-423020100002 00005

2. World Health Organization (WHO). Who Report on the Tobacco Epidemic, 2011 Warning about the dangers of tobacco. Suíça, 2011 [acessado 2017 Set 13]. Disponível em: http://www.who.int/tobacco/global_report/2011/exec_summary/en/

3. Organização Mundial da Saúde (OMS). Classificação Estatística Internacional de Doenças e Problemas Relacionados à Saúde - CID-10. 2008. [acessado 2017 Set 13]. Disponível em: http://www.cid10.com.br

4. American Psychiatric Association (APA). Diagnostic and statistical manual of mental disorders: DSM-5. $5^{\text {th }}$ ed. Washington: Artmed; 2014.

5. Brasil. Ministério da Saúde (MS). Pesquisa Nacional de Saúde - PNS 2013: percepção do estado de saúde, estilos de vida e doenças crônicas. Brasília: MS; 2014. [acessado 2017 Set 13]. Disponível em: http://portalarquivos.saude.gov.br/images/pdf/2015/agosto/24/ PNS-Volume-1-completo.pdf

6. Ng M, Freeman KM, Fleming DT, Robinson M, Dwyer-Lindgren L, Thomson B, Wollum A, Sanman E, Wulf S, Lopez AD, Murray CJ, Gakidou E. Smoking Prevalence and Cigarette Consumption in 187 Countries, 1980-2012. JAMA 2014; 311(2):183-192.

7. Kabwama SN, Ndyanabangi S, Mutungi G, Wesonga R, Bahendeka SK, Guwatudde D. Tobacco use and associated factors among Adults in Uganda: Findings from a nationwide survey. Tob Induc Dis 2016; 14:27.

8. Uguru NP, Mbachu C, Ibe OP, Uguru CC, Odukoya $\mathrm{O}$, Okwuosa $\mathrm{C}$, Onwujekwe O. Investigating male tobacco use and expenditure patterns across socio-economic groups in Nigeria. PLoS One 2015; 10(4):e0122021.

9. Cullen J, Mowery P, Delnevo C, Allen JA, Sokol N, Byron MJ, Thornton-Bullock A. Seven-year patterns in US cigar use epidemiology among young adults aged 18-25 years: a focuson race/ethnicity and brand. Am J Public Health 2011; 101(10):1955-1962.

10. Richardson A, Xiao H, Vallone DM. Primary and dual users of cigars and cigarettes: profiles, tobacco use patterns and relevance to policy. Nicotine Tob Res 2012; 14(8):927-932.

11. Berg CJ, Ajay VS, Ali MK, Kondal D, Khan HM, Shivashankar R, Pradeepa R, Mohan D, Fatmi Z, Kadir MM, Tandon N, Mohan V, Narayan KM, Prabhakaran D. A cross-sectional study of the prevalence and correlates of tobacco Use in Chennai, Delhi, and Karachi: data from the CARRS study. BMC Public Heath 2015; 15(483):1-12.

12. Singh A, Ladusingh L. Prevalence and determinants of tobacco use in India: evidence from recent Global Adult Tobacco Survey data. PLoS One 2014; 9(12):e114073.

13. Abreu MN, Caiaffa WT. Influência do entorno familiar e do grupo social no tabagismo entre jovens brasileiros de 15 a 24 anos. Rev Panam. Salud Publica 2011;30(1):22-30. 
14. Rodrigues LC, Werneck GL. Estudos de caso controle. In: Medronho RA, Bloch KV, Luiz RR, Werneck GL, organizadores. Epidemiologia. $2^{a}$ ed. São Paulo: Atheneu; 2009. p. 221-236.

15. Barreto SM, Giatti L, Oliveira-Campos M, Andreazzi MA, Malta DC. Experimentation and use of cigarette and other tobacco products among adolescents in the Brazilian state capitals (PeNSE 2012). Rev Bras Epidemiol 2014; 17(Supl. 1):62-76.

16. Hamilton LC. Statistics with STATA: Version $12.8^{\text {th }}$ ed. Boston: Cengage Learning; 2013.

17. Carmo JT, Pueyo AA. A adaptação ao português do Fagerström test for nicotine dependence (FTND) para avaliar a dependência e tolerância à nicotina em fumantes brasileiros. Rev Bras Med 2002; 59(1):73-80.

18. Reichert J, Araújo AJ, Gonçalves CM, Godoy I, Chatkin JM, Sales MP, Oliveira MVC, Castillo PL, Meirelles RHS. Diretrizes para cessação do tabagismo. J Bras Pneumol 2004 [acessado 2017 Set 13]; 30:1-75. Disponível em: http://www.scielo.br/pdf/jbpneu/v30s2/ a02v30s2.pdf

19. Smilkstein G. The family APGAR: a proposal for a family function test and its use by physicians. J Fam Pract 1978; 6(6):1231-1239.

20. Duarte Y. Família: rede de suporte ou fator estressor: a ótica de idosos e cuidadores familiares [tese]. São Paulo: Universidade de São Paulo; 2001.

21. Méndez EB. Uma Versão Brasileira do AUDIT (Alcohol Use Disorders Identification Test) [dissertação]. Pelotas: Universidade Federal de Pelotas; 1999.

22. Lima CT, Freire AC, Silva AP, Teixeira RM, Farrell $\mathrm{M}$, Prince $\mathrm{M}$. Concurrent and construct validity of the AUDIT in an urban Brazilian sample. Alcohol Alcohol 2005; 40(6):584-589.

23. Porta MA. Dictionary of Epidemiology. $6^{\text {th }}$ ed. Oxford: Oxford University Press; 2014. [acessado 2017 Set 13] Disponível em: http://irea.ir/files/site1/pages/dictionary.pdf

24. Diez-Roux AV. Multilevel analysis in public health research. Annu Rev Public Health 2000; 21:171-192.

25. Brasil. Ministério da Saúde (MS). Conselho Nacional de Saúde. Resolução no 466, de 12 de dezembro de 2012. Diário Oficial da União 2013; 13 dez.

26. Vargas LS, Lucchese R, Silva AC, Guimarães RA, Vera I, Castro PA. Determinants of tobacco use by students. Rev Saude Publica 2017; 51(36):1-9.

27. Rubinstein ML, Shiffman S, Rait MA, Benowitz NL. Race, gender and nicotine metabolism in adolescent smokers. Nicotine Tob Res 2013; 15(7):1311-1315.

28. Branstetter SA, Muscat JE. Time to first cigarette and serum cotinine levels in adolescent smokers: National Health and Nutrition Examination Survey, 20072010. Nicotine Tob Res 2013; 15(3):701-707.

29. Dempsey DA, Meyers MJ, Oh SS, Nguyen EA, Fuentes-Afflick E, Wu AH, Jacob P, Benowitz NL. Determination of tobacco smoke exposure by plasma cotinine levels in infants and children attending urban public hospital clinics. Arch Pediatr Adolesc Med 2012; 166(9):851-856.

30. Malta DC, Vieira ML, Szwarcwald L, Caixeta R, Briro SM, Reis AA. Tendências de tabagismo entre a população brasileira - Pesquisa Nacional de Agregados Familiares, 2008 e Pesquisa Nacional de Saúde, 2013. Rev Bras Epidemiol 2015; 18(2):45-56.
31. Müller EV, Silva MT, Grden CR, Reche PM, Borges PK, Sousa JA. Fatores associados ao tabagismo em usuários da estratégia saúde da família. Cogitare Enferm 2017; 22(4):1-10.

32. Instituto Brasileiro de Geografia e Estatística (IBGE). Pesquisa Nacional por Amostra de Domicílios: Sintese de indicadores sociais: uma análise das condições de vida da população brasileira. Rio de Janeiro: IBGE; 2017.

33. Panaino EF, Soares CB, Campos CM. Contextos de início do consumo de tabaco em diferentes grupos sociais. Rev Latino-Am Enfermagem 2014; 22(3):379385.

34. Grills NJ, Singh R, Singh R, Martin BC. Tobacco Usage in Uttarakhand: A Dangerous Combination of High Prevalence, Widespread Ignorance, and Resistance to Quitting. Biomed Res Int 2015; 2015:132120.

35. Berg CJ, Zheng P, Kegler MC. Family interactions iegarding fathers' smoking and cessation in Shanghai, China. J Smok Cessat, 2014; 11(4)199-202.

36. Rathod SD, Nadkarni A, Bhana A, Shidhaye R. Epidemiological features of alcohol use in rural India: a population-based cross-sectional study. BMJ Open 2015; 5(12):e009802.

37. Kruse GR, Bangsberg DR, Hahn JA, Haberer JE, Hunt PW, Muzoora C, Bennett JP, Martin JN, Rigotti NA. Tobacco use among adults initiating treatment for HIV infection in rural Uganda. AIDS Behav 201; 18(7):1381-1389

38. Lakew Y, Haile D. Tobacco use and associated factors among adults in Ethiopia: further analysis of the 2011 Ethiopian Demographic and Health Survey. BMC Public Health 2015; 15:487.

39. Kahar P, Misra R, Patel TG. Sociodemographic Correlates of Tobacco Consumption in Rural Gujarat, India. Biomed Res Int 2016; 2016:5856740.

40. Instituto Brasileiro de Geografia e Estatística (IBGE). Pesquisa Nacional por Amostra de Domicílios: Um panorama da saúde no Brasil. Acesso e utilização dos serviços, condições de saúde e fatores de risco e proteção em saúde 2008. Rio de Janeiro: IBGE; 2010.

41. Malta DC, Moura EC, Oliveira M, Santos FP. Usuários de planos de saúde: morbidade e uso de exames preventivos, por inquérito telefônico, Brasil, 2008. Cad Saude Publica 2011; 27(1):57-66.

42. Woodgate RL, Kreklewetz CM. Youth's narratives about family members smoking: parenting the parent- it's not fair! BMC Public Health 2012; 12:965.

43. Nogueira AL, Sousa F G, Silva IR, Silva AC Silva DC, Santana E. Funcionalidade de famílias de adolescentes em escolas públicas: uma abordagem descritiva. Cogitare Enfermagem 2012; 17(2):3224-3117.

44. Soto KE, Carrillo MH, Cassiani CA, Munevar AM, Cardona MC. Factores relacionados con el consumo de sustancias psicoactivas en una institución educativa de Jamundí Valle, Colômbia. Rev Colomb Psiquiat 2016; 45(1):2-7.

45. Selya AS, Dierker LC, Rose JS, Hedeker D, Mermelstein RJ. Risk factors for adolescent smoking: parental smoking and the mediating role of nicotine dependence. Drug Alcohol Depend 2012; 124(3):311-318.

46. Park S, Kim Y. Prevalence, correlates, and associated psychological problems of substance use in Korean adolescentes. BMC Public Health 2016; 16:79. 
47. Berge J, Sundell K, Öjehagen A, Håkansson A. Role of parenting styles in adolescent substance use: results from a Swedish longitudinal cohort study. BMJ Open 2016; 6(1):1-9.

48. Goodwin RD, Pagura J, Spiwak R, Lemeshow AR, Sareen J. Predictors of persistent nicotine dependence among adults in the United States. Drug Alcohol Depend 2011; 118(2-3):127-133.

49. Flensborg-Madsen T1, von Scholten MB, Flachs EM, Mortensen EL, Prescott E, Tolstrup JS. Tobacco smoking as a risk factor for depression. A 26year population-based follow-up study. J Psychiatr Res 2011; 45(2):143-149.

50. Bakar C, Gündogar D, Karaman HI, Maral I. Prevalence and related risk factors of tobacco, alcohol and illicit substance use among university students. Eur $J$ Psychiat 2013; 27(2):97-110.

51. Piwoński J, Piwońska A, Sygnowska E. Do depressive symptoms adversely affect the lifestyle? Results of the WOBASZ study. Kardiol Pol 2010; 68(8):912-918.

52. Gomes FC, Andrade AG, Izbicki R, Moreira Almeida A, Oliveira LG. Religion as a protective factor against drug use among Brazilian university students: a national survey. Rev Bras Psiquiatr 2013; 35(1):29-37.

53. Bailey ZD, Slopen N, Albert M, Williams DR. Multidimensional religious involvement and tobacco smoking patterns over 9-10 years: A prospective study of middle-aged adults in the United States. Soc Sci Med 2015; 138:128-135

54. Becker J, Schaub MP, Gmel G, Haug S. Cannabis use and other predictors of the onset of daily cigarette use in young men: what mattersmost? Results from a longitudinal study. BMC Public Health 2015; 15:843

55. Byron MJ, Cohen JE, Gittelsohn J, Frattaroli S, Nuryunawati R, Jernigan DH. Infhuence of religious organisations' statements on compliance with a smokefree law in Bogor, Indonesia: a qualitative study. $B M J$ Open 2015; 5(12):e008111.

56. Agrawal A, Madden PA, Martin NG, Lynskey MT. Do early experiences with cannabis vary in cigarette smokers? Drug Alcohol Depend 2013; 128(3):255-259.
57. Do EK, Prom-Wormley EC, Eaves LJ, Silberg JL, Miles DR, Maes HH. Genetic and Environmental Influences on Smoking Behavior across Adolescence and Young Adulthood in the Virginia Twin Study of Adolescent Behavioral Development and the Transitions to Substance Abuse Follow-Up. Twin Res Hum Genet 2015; 18(1):43-51.

58. Korhonen T, Levälahti E, Dick DM, Pulkkinen L, Rose RJ, Kaprio J, Huizink AC. Externalizing behaviors and cigarette smoking as predictors for use of illicit drugs: a longitudinal study among Finnish adolescent twins. Twin Res Hum Genet 2010; 13(6):550-558.

59. Yang JJ, Song M, Yoon HS, Lee HW, Lee Y, Lee SA, Choi JY, Lee JK, Kang D. What Are the Major Determinants in the Success of Smoking Cessation: Results from the Health Examinees Study. PLoS One 2015; 10(12):e0143303.

60. Sanjuan PM, Rice SL, Witkiewitz K, Mandler RN, Crandall C, Bogenschutz MP. Alcohol, tobacco, and drug use among emergency department patients. Drug Alcohol Depend 2014; 138:32-38

61. Benowitz N L. Nicotine addiction. N Engl J Med 2010; 362(24):2295-2303.

62. Doyon WM, Thomas AM, Ostroumov A, Dong Y, Dani JA. Potential substrates for nicotine and alcohol interactions: a focus on the mesocorticolimbicdopamine system. Biochem Pharmacol 2013; 86(8):11811193.

Artigo apresentado em 13/10/2017

Aprovado em 07/04/2018

Versão final apresentada em 09/04/2018 